\title{
Allocation of Book Funds in College Libraries
}

Mr. Richards is librarian, Carleton College, Northfield, Minn.

$\mathrm{A}^{\mathrm{L}}$ CATION of book funds to academic subject fields is still of widespread interest among college librarians. Theoretical and practical reports have been published. ${ }^{1}$ In a study of recent practice in twelve colleges at the end of the year $195 \mathrm{I}$ -

1 Randall, William M. "The College-Library Book Budget." Library Quarterly, I :42 I $=435$, Oct. I93 I.

Reeves, Floyd W. and Russell, John D. "The Administration of the Library Budget." Library Quarterly,

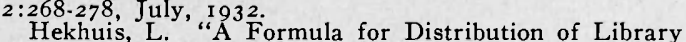
Funds among Departments." Library Journal, 6i :574575, Aug. 1936 .

Muller, Hans. "The Management of College Library Book Budgets." College and Research Libraries, 2:320326, Sept. I 941

Coney, Donald. "An Experimental Index for Apportioning Departmental Book Funds for a University
1952 some interesting new data were compiled and are presented below. The three tables represent parts of three distinct phases of the allocation problem. The first shows the percentage of allocated funds allowed each department in each of nine college libraries. (Three of the twelve reported that they do not allocate.) This then is a sinall cross section of what was being done in 195I-1952. The second table shows a new method for determining one of the factors significant in the allocation formula.

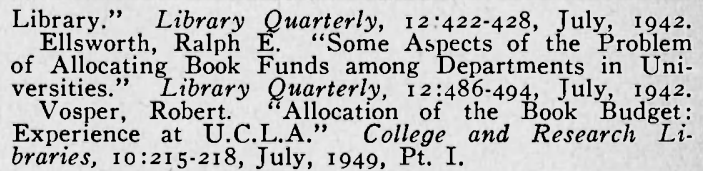

Library." Library Quarterly, I 2:422-428, July, I942. Ellsworth, Ralph E. "Some Aspects of the Problem versities." Library Ouarterly, I $2: 486-494$, July, I042. Vosper, Robert. "Allocation of the Book Budget: braries, I0:2 I 5-2 I 8, July, I 949, Pt. I.

TABLE I

Percentage of Allocated Funds Allowed Each Department in Nine Allocating College Libraries During I 95 I-I 952

\begin{tabular}{|c|c|c|c|c|c|c|c|c|c|}
\hline Department ${ }^{1}$ & I & 2 & 3 & 4 & 5 & 6 & 7 & 8 & 9 \\
\hline Art & 2.33 & $4 \cdot 54$ & 6.12 & 5.07 & 4.03 & $3 \cdot 3^{2}$ & 5.10 & 6.65 & 2.95 \\
\hline Biology & 5.24 & $3 \cdot 3 \mathrm{I}$ & $5 \cdot 44$ & 8.45 & 8.06 & 5.22 & $7 \cdot 40$ & $9 \cdot 76$ & 5.88 \\
\hline Chemistry & 2.09 & 2.07 & $5 \cdot 44$ & 4.87 & 4.03 & 3.08 & 7.00 & 21.20 & 5.88 \\
\hline Classics. & I. 3 I & & 2.03 & & $2.8 \mathrm{I}$ & .47 & 2.50 & 3.05 & 3.68 \\
\hline Economics & 5.24 & $7 \cdot 44$ & $4 \cdot 76$ & $5 \cdot 90$ & 7.25 & $9 \cdot 7^{2}$ & $9 \cdot 40$ & $3 \cdot 4^{2}$ & 8.82 \\
\hline English & 17.05 & I $5 \cdot 70$ & $9 \cdot 53$ & 14.29 & $14.9 \mathrm{I}$ & 12.09 & 8.90 & 6.34 & I 4.7 I \\
\hline Geology & 6.05 & $3 \cdot 30$ & I. 35 & 4.23 & & 4.03 & & $3 \cdot 54$ & \\
\hline German & .66 & 1.65 & 4.08 & 2.28 & 3.09 & I. 42 & 4.60 & I. 16 & 2.94 \\
\hline Gov't. \& I.R. & $4 \cdot 50$ & $7 \cdot 44$ & 4.08 & $5 \cdot 15$ & 4.03 & 9.00 & $9 \cdot 36$ & 2.69 & 8.82 \\
\hline History & $4 \cdot 5 \mathrm{I}$ & 19.01 & 12.94 & $7 \cdot 76$ & 10.08 & $9 \cdot 48$ & 14.80 & 5.85 & 8.82 \\
\hline Math. \& Ast. & 4.18 & $3 \cdot 3 \mathrm{I}$ & $3 \cdot 40$ & I. 78 & 4.03 & 2.37 & $5 \cdot 30$ & .56 & $4 \cdot 4 \mathrm{I}$ \\
\hline Music & 6.58 & 2.89 & 6.80 & 3.24 & 2.82 & 2.84 & .62 & $4 \cdot 46$ & 3.68 \\
\hline Phil. \& Religion & I 4.45 & $4 \cdot 95$ & 14.98 & $9 \cdot 4^{8}$ & 7.25 & $7 \cdot 58$ & 8.10 & 7.10 & 8.82 \\
\hline Physics & 2.62 & 2.07 & 4.08 & $2.4 \mathrm{I}$ & 4.03 & I. I 8 & 3.18 & $3 \cdot 72$ & 5.88 \\
\hline Phys. Education & 2.09 & $4 \cdot 96$ & 4.08 & 2.66 & 2.82 & 2.84 & & $4 \cdot 94$ & \\
\hline Psych. \& Ed. & 9.18 & 8.26 & $4 \cdot 76$ & 8.69 & IO. 48 & 12.80 & 8.80 & 8.72 & 5.88 \\
\hline Romance Lang. & I. 3 I & $3 \cdot 3 \mathrm{I}$ & 6.13 & $4 \cdot 55$ & 6.18 & $3 \cdot 32$ & $4 \cdot 94$ & 2.33 & 5.88 \\
\hline $\begin{array}{l}\text { Sociology } \\
\text { Percentage of total book }\end{array}$ & $6.5^{8}$ & $5 \cdot 79$ & 4.08 & 9.19 & 4.03 & 9.24 & & $4 \cdot 5^{2}$ & 2.95 \\
\hline fund allocated & $4 \mathrm{I}$ & 86 & 68 & 65 & 89 & 38.7 & 62.5 & & 68.8 \\
\hline
\end{tabular}

I All colleges do not group their courses under identical departmental headings. It was necessary to take some liberties to separate or combine them into a uniform list. Where this was done an attempt was made to determine the proper proportions by studying the announcement of courses published by the reporting institution. 
TABLE II

Average Cost of Books by Subject Field

\begin{tabular}{|c|c|c|c|c|}
\hline Department & $\begin{array}{l}\text { Total } \\
\text { Net } \\
\text { Cost }\end{array}$ & $\begin{array}{l}\text { No. } \\
\text { Units }\end{array}$ & $\begin{array}{c}\text { Average } \\
\text { Net } \\
\text { Cost }\end{array}$ & $\begin{array}{l}\text { Adjusted } \\
\% \text { of } \\
\text { Total }\end{array}$ \\
\hline Art & $\$ 94 \mathrm{I} .68$ & I 49 & $\$ 6.32$ & a \\
\hline Biography & $134 \cdot 4^{2}$ & 47 & 2.86 & $3 \cdot 77$ \\
\hline Botany & 163.13 & 34 & 4.80 & 6.33 \\
\hline Chemistry & 221.68 & 27 & $8.2 \mathrm{I}$ & I0.83 \\
\hline Classics & II 10.69 & 27 & 4.10 & $5.4 \mathrm{I}$ \\
\hline Economics & 297.90 & 86 & $3 \cdot 46$ & 4.57 \\
\hline English & 423.55 & 135 & 3.14 & 4.15 \\
\hline Geology & 177.53 & $3 \mathrm{I}$ & $5 \cdot 73$ & $7 \cdot 56$ \\
\hline German & 76.90 & 17 & 4.52 & $5 \cdot 96$ \\
\hline Gov't. \& I.R. & 364.30 & 102 & $3 \cdot 57$ & \\
\hline History & 545.84 & I IO & 4.96 & 6.55 \\
\hline Math. \& Ast. & 172.42 & 37 & 4.66 & 6.15 \\
\hline Music & 95.02 & $2 \mathrm{I}$ & $4 \cdot 52$ & $5 \cdot 96$ \\
\hline Phil. \& Religion & $166.6 \mathrm{I}$ & 47 & $3 \cdot 54$ & 4.67 \\
\hline Physical Ed. & 94.25 & 24 & 3.93 & \\
\hline Physic & $\mathrm{I} 24.4 \mathrm{I}$ & 26 & $4 \cdot 78$ & $6.30^{-}$ \\
\hline Psych. \& Ed. & 229.14 & 50 & $4 \cdot 58$ & 6.04 \\
\hline Romance Lang. & $137 \cdot 37$ & 43 & 3.19 & $4.2 \mathrm{I}$ \\
\hline Sociology & 247.22 & 63 & $3 \cdot 9^{2}$ & $5 \cdot 18$ \\
\hline Zoology & 216.90 & 45 & 4.82 & $6 \cdot 36$ \\
\hline Total & & & $\$ 89.6 \mathrm{I}$ & 0.00 \\
\hline
\end{tabular}

The third table shows the application of Table II and one other factor to the problem in one institution. The result, in terms of dollars, was a combination of the formula and a reasoned, frank discussion of local emphasis, aims, and lacunae. The figures were "rounded off" in the process.

TABLE III

Allocation at Carleton Based on Cost Factor and Enrolment Distribution

\begin{tabular}{|c|c|c|c|c|c|c|}
\hline Department & $\begin{array}{l}\text { Enrolment } \\
\text { Index }\end{array}$ & $\begin{array}{l}\text { Cost } \\
\text { Index }\end{array}$ & $\begin{array}{c}\text { Âverage } \\
\text { Cost- } \\
\text { Enrolment }\end{array}$ & $\begin{array}{l}\text { Preliminary } \\
\text { Allocation }\end{array}$ & $\begin{array}{l}\text { Average } \\
\text { Expendi- } \\
\text { tures } \\
1946-52^{2}\end{array}$ & $\begin{array}{c}\text { Final } \\
\text { Allocation } \\
\text { fior } \\
\text { I } 952-3\end{array}$ \\
\hline \multicolumn{7}{|l|}{$\mathrm{Art}^{3}$} \\
\hline Biography & $3 \cdot 73$ & $3 \cdot 77$ & $3 \cdot 75$ & I 29.75 & I $21 . \infty$ & I 10.00 \\
\hline Botany & 2.34 & 6.33 & $4 \cdot 34$ & I 50.16 & I 5 I . 00 & I 50.00 \\
\hline Chemistry & $3 \cdot 75$ & 10.83 & 7.29 & 252.23 & $2 \mathrm{I}_{4} . \infty$ & 240.00 \\
\hline Classics & I. .08 & $5 \cdot 4 \mathrm{I}$ & 3.24 & I I 2.10 & $95 . \infty 0$ & 100.00 \\
\hline Economics & $4 \cdot 49$ & $4 \cdot 57$ & $4 \cdot 53$ & I 56.74 & 238.00 & 200.00 \\
\hline English & $17 \cdot 44$ & 4.15 & 10.80 & 373.68 & 431.00 & 395.00 \\
\hline Geology & $3.8 \mathrm{I}$ & $7 \cdot 56$ & 5.68 & 196.52 & I 55.00 & 170.00 \\
\hline \multicolumn{7}{|l|}{ Gov't. \& I.R. ${ }^{3}$} \\
\hline History & 10.87 & 6.55 & $8.7 \mathrm{I}$ & $301 \cdot 36$ & 534.00 & 430.00 \\
\hline Math. \& Ast. & $5 \cdot 56$ & 6.15 & 5.85 & $202.4 \mathrm{I}$ & I 57.00 & 200.00 \\
\hline Music & 6.70 & 5.96 & 6.33 & 219.02 & 145.00 & 170.00 \\
\hline \multicolumn{7}{|l|}{ Phys. Education ${ }^{3}$} \\
\hline Physics & I. 88 & 6.30 & 4.09 & I 4 I. $5 \mathrm{I}$ & 105.00 & 140.00 \\
\hline Psych. \& Ed. & 5.66 & 6.04 & 5.85 & $202.4 \mathrm{I}$ & 278.00 & 200.00 \\
\hline Romance Languages & 9.06 & $4.2 \mathrm{I}$ & 6.64 & $229 \cdot 74$ & $245.00^{4}$ & 215.00 \\
\hline Sociology & $7 \cdot 33$ & 5.18 & 6.26 & 216.59 & 206.00 & 210.00 \\
\hline \multirow[t]{2}{*}{ Zoology } & 5.22 & 6.36 & $5 \cdot 79$ & $200 \cdot 33$ & 216.00 & 200.00 \\
\hline & 100.00 & 100.00 & 100.00 & $3,459 \cdot 96$ & & $3,460.00^{:}$ \\
\hline
\end{tabular}

1 Not including any postage charges, i.e. this is entirely for books.

2 Includes postage pro-rated to each book purchased.

3 Omitted-see note, Table II.

4 In part "inflated" by special gifts of several hundred dollars on two occasions.

$56.5 \%$ of all book funds excepting endowment and grant funds, and postage.

For reasons in part peculiar to Carleton, the determination of the allocalion pallern was initially based entirely on the cost factor (Table II) and enrolment distribution. Experimentally postage was also separated out of the entire book budget at the start. The preliminary allocation was then considered by the Faculty Library Commillee in the light of the previous expenditures, $1946-$ 52, and the practice in comparable schools elsewhere as shown in Table I. Special circumstances affecting Carleton were discussed and a frank bu! admiltedly subjective appraisal of the book collection entered considerations before the final allocation was formulated. 